\title{
REMEMORAÇÃO, SUBJETIVIDADE E AS BASES NEURAIS DA MEMÓRIA AUTOBIOGRÁFICA
}

\author{
Jean Frank* \\ J. Landeira-Fernandez ${ }^{* *}$
}

\section{Resumo}

Nesta revisão abordamos o sistema de memória autobiográfica como um processo de rememoração subjetiva graças à ativação de um substrato neural próprio para esta qualidade mnemônica. A rememoração autobiográfica recruta vias corticais extensas tendo como ponto de convergência a região frontal e suas interconexões, culminando com a área orbitofrontal. Trata-se de um processamento neural complexo capaz de integrar diferentes aspectos da evocação, tais como auto-identidade, controle, seletividade e emoção.

Analisamos também a noção de amnésia orgânica e amnésia funcional com base em achados recentes sobre os efeitos do estresse no cérebro. Dentre estas evidências, destacamse as alterações morfológicas e neuroquímicas no cérebro produzidas por estímulos estressantes assim como o alívio destes efeitos através da psicoterapia. Este conhecimento representa um avanço considerável nos conceitos de psicopatologia, abrindo caminhos para a compreensão das bases neurais da personalidade.

Palavras-chave: memória, córtex associativo, subjetividade, neuropsicologia

\section{AbSTRACT}

REMEMBERING, SUBJECTIVITY AND THE NEURAL BASIS OF THE AUTOBIOGRAPHIC MEMORY

This review addresses autobiographical memory as a subjective process supported by a neural system appropriate for a personal recall capacity. Autobiographical recall activates extensive

* Professor do Departamento de Psicologia, PUC-Rio, Instituto de Neurologia de Goiânia, Bolsista de pós-doutorado do CNPq.

** Professor do Departamento de Psicologia, PUC-Rio, Curso de Psicologia Universidade Estácio de Sá, Bolsista de produtividade do CNPq. 
cortical pathways that converge to the frontal region and its interconnections culminating in the orbitfrontal area. It is a complex neuronal system capable of integrating different aspects of recall such as: self-identity, control, selectivity and emotion. We also analyze the notion of organic amnesia and functional amnesia based on recent findings of changes in the brain related to stress and psychological trauma. Among these evidences, we highlight the morphological and neurochemical disturbances in the brain caused by stressful stimuli. These data and concepts represent a considerable advancement regarding concepts of psychopathology and opens avenues for the understanding of the neurological bases of personality.

Keywords: memory, cortex, subjectivity, neuropsychology

\section{INTRODUÇÃO}

A evolução dos conhecimentos sobre a relação mente-cérebro nas últimas décadas atingiu os vários domínios do saber que tratam da questão do comportamento, personalidade e psicopatologia (Halligan e David, 2001). Como uma chave para a abertura de novos caminhos, os estudos sobre mente e cérebro são muito promissores. São sedutores por desvendar mistérios encerrados no corpo humano e suas possíveis relaçôes com a nossa atividade mental. Acenam com a possibilidade do sempre buscado controle sobre processos complexos por introduzir uma abordagem por vezes considerada mais objetiva, advinda das ciências biológicas. E, mais ainda, oferecem ao sujeito a oportunidade de ser objeto de si próprio ao abordar a mente como algo externo a ser observado, um órgão do corpo para ser analisado e investigado.

A evocação da informação autobiográfica é um processamento significativo para a noção de auto-identidade. Lembramos os detalhes da nossa história pessoal com todo o seu colorido emocional associado a imagens com simbologia própria. Esse sistema permite também a permanência e continuidade do eu através do tempo e, principalmente, a adaptação da pessoa ao seu meio interior e exterior. $\mathrm{O}$ esquecimento, por outro lado, oferece ao indivíduo a oportunidade de selecionar e de adequar-se aos eventos que em determinado momento lhe são mais importantes. Esquecer e lembrar são processos centrais nas psicoterapias dinâmicas, pois refletem o acervo pessoal. Neste artigo, vamos nos valer da análise dos dados da neuropsicologia da memória autobiográfica para abrir caminhos visando a compreensão da relação mente-cérebro como um fenômeno configurado em relação à consciência do eu resultante da experiência pessoal e com significado próprio para o sujeito. 
Abordaremos dois aspectos da neuropsicologia da memória autobiográfica por sua repercussão sobre a subjetividade, sendo estes: i) análise das bases neurais da memória autobiográfica como uma representação do passado pessoal; ii) consideraçôes, à luz das investigaçôes com neuroimagem funcional, da neuropsicologia da memória autobiográfica e da amnésia retrógrada focal. Abriremos a exposição com conceitos fundamentais sobre os múltiplos sistemas de memória para em seguida destacar a importância da memória autobiográfica na origem de processos subjetivos.

\section{Os MÚLTIPLOS SISTEMAS DE MEMÓRIA}

A noção de memória tem uma representação popular marcante, particularmente por sua propriedade de tornar o passado presente. Para fins da proposta deste artigo, conceituaremos a memória como sendo um processo cognitivo estruturado por um conjunto de operaçōes que respondem a regras de integração com o meio ambiente com substratos neurais específicos. Este processo de interação resulta no registro, permanente ou não, de uma experiência através do tempo e em mudança no comportamento relativamente duradoura. Permite a continuidade do indivíduo, preservando sua identidade, ao longo das variações que compõem as experiências do cotidiano (Frank, 2002).

A memória pode ser dividida em relação ao tempo e ao conteúdo (Markowitsch, 2003). Em geral, a dimensão temporal é entendida como memória de curtíssimo prazo, memória de curto prazo, memória de longo prazo e memória de longuíssimo prazo. A memória de curtíssimo prazo é responsável pelo armazenamento muito rápido (na ordem de alguns milissegundos), geralmente com uma representação auditiva ou visual (Cowan, 1996). A informação na memória de curto prazo é mais duradoura (na ordem de minutos), permitindo a realização de trabalho mais elaborado sobre a informação inicialmente codificada. A memória operacional, conforme descrita por Baddely (2000), é um tipo de memória de curto prazo que dá condição para a manipulação de informações armazenadas de forma consciente. Exemplos clássicos da memória operacional são a permanência da informação para o trabalho de decisão rápida como em certos tipos de escolha, realização de cálculo mental, aplicação de regras para resolver problemas. É o componente do sistema de informação humano que permite ao indivíduo manter-se consciente ao longo dos eventos e das demandas múltiplas na interação com o meio. A memória de longo prazo está relacionada com informaçōes que foram consolidadas e armazenadas por um período de tempo bem mais longo (na ordem de dias e meses). Estas informaçôes são consolidadas dentro de uma janela de tempo e ar- 
mazenadas nos córtices de associação de acordo com as suas propriedades e modalidades (McGaugh, 2000). Finalmente, memórias de longuíssimo prazo podem ficar armazenadas por um período de tempo quase que ilimitado. São informaçôes que foram consolidadas e podem ficar armazenadas por praticamente toda uma vida.

A divisão da memória segundo o conteúdo refere-se à organização das informaçôes hierarquicamente estruturadas em relação ao momento e ao local ou contexto onde foram adquiridas (Markowitsch, 1999; Tulving, 1987). Neste sentido, a memória pode ser episódica ou semântica. A memória episódica refere-se ao passado, sendo mais específica em termos do contexto e do tempo, ou seja, onde e quando. Atravessa o tempo, permitindo a continuidade da identidade individual, a evocação de fatos da história pessoal, como na memória autobiográfica. A memória semântica é voltada para o presente e contém o acervo de fatos e informações sobre o mundo do indivíduo, desde o conjunto de conhecimentos sobre sua linguagem, vocabulário, regras de gramática, como conceitos e significados diversos. É um acervo dinâmico de informações, mas no geral não requer a evocação temporal e de detalhes contextuais. É muito duradoura e bem mais refratária a disfunções corticais. Exemplificando, os relatos sobre um determinado acidente de automóvel são dados episódicos, já a explicação sobre acidentes de automóveis é uma informação de teor semântico e menos rica de evocação episódica. As fronteiras entre estes sistemas de memória são sujeitas a mudanças pela vontade do sujeito. Por exemplo, podemos iniciar um relato com dados mais semânticos, presentes, e dar continuidade evocando instâncias específicas que requerem um acesso episódico aos dados do passado. Portanto, memória autobiográfica tem elementos semânticos, como aqueles que se referem à noção do eu, e aspectos episódicos como a rememoração de fatos contextuais (datas, faces, nomes, detalhes, locais) da experiência do sujeito.

Há ainda todo um sistema de memória que independe de processamento consciente. Destaca-se aí a memória perceptual, que se refere ao julgamento de familiaridade, o fenômeno de pré-ativação ou "priming" que permite o processamento não consciente de informações anteriormente percebidas, e a memória de procedimento, que é responsável pelo armazenamento de habilidades sensóriomotoras (Markowitsch, 2003). Estes tipos de memória, segundo a taxonomia avançada por Endel Tulving (1987), não serão alvo deste artigo por fugirem à temática proposta.

A concepção da memória como um fenômeno neurocognitvo estruturado por múltiplos sistemas oferece uma base conceitual importante para os dados clínicos nos casos de amnésia. Em geral, observamos uma dissociação entre os 
tipos de amnésia, sendo comum a debilidade da memória recente com preservação da memória remota. Esta dissociação indica a existência de circuitos neurais específicos em relação aos diferentes sistemas de memória. Outros fenômenos de dissociação têm sido observados na investigação clínica como o esquecimento autobiográfico com a memória anterógrada preservada, usualmente denominado de amnésia retrógrada focal. São os casos de amnésia acompanhados ou não de outras alterações cognitivas e de personalidade (Markowitsch, 2003). Estaremos abordando esta classe de amnésia mais adiante neste artigo.

A memória autobiográfica é uma evocação episódica de datas, eventos e incidentes pessoais através do tempo e da relação espacial e temporal entre eles (Greenberg e Rubin 2003). É mais do que uma mera evocação de fatos do passado, pois a pessoa que lembra tem consciência da evocação como uma experiência da sua história pessoal. Como realçam Greenberg e Rubin, a memória autobiográfica consiste numa rememoração com a consciência de que o fato ocorreu anteriormente consigo próprio. Neste sentido, é um processo de memória que combina elementos de memória semântica e memória episódica. Envolve a memória semântica, pois é necessário primeiramente um conhecimento de si próprio para que os dados tenham significado como pertencentes ao indivíduo em particular. Incorpora também a qualidade episódica por serem narrativas pessoais de situações, nomes, faces que foram vividos, conhecidos pelo sujeito no seu passado.

Greenberg e Rubin (2003), apoiados por autores da área, distinguem entre a evocação de fatos autobiográficos, que são dados sobre a história pessoal, e a memória autobiográfica propriamente dita, relacionada com a evocação de informações acompanhada da sensação de rememoração dos fatos como parte da consciência do eu. Esta experiência de rememoração enfatiza a natureza da emoção que acompanha a consciência da evocação autobiográfica. Neste caso, a memória autobiográfica é acompanhada por um estado emocional próprio de familiaridade com o contexto e com o momento em que estas informações foram adquiridas. Possivelmente a integração temporo-espacial entre os dados da memória autobiográfica seja facilitada pela experiência emocional de familiaridade com o tom de pertencimento ao eu. Em casos de demências, conforme a patologia invade o córtex, começam a surgir falhas no processo de evocação de informações remotas. Estas distorções da realidade passada podem ocorrer sobre a rememoração do passado pessoal. São fatos do passado pessoal evocados com a qualidade de rememoração confabulada provavelmente por falhas nas vias cerebrais frontotemporais responsáveis pela facilitação do acesso a informações remotas. Há falha tanto na qualidade do dado evocado, como no colorido emocional que lhe é auferido, pois ocorre um falso sentimento de rememoração dos fatos. 


\section{BASES NEURAIS DA MEMÓRIA AUTOBIOGRÁFICA}

Este é um tema efervescente na área de neurociências. Faremos referência a noções já bem estabelecidas na literatura. O conceito de sistemas de memória, conforme descrito acima, advém da psicologia cognitiva, tendo oferecido bases conceituais para a compreensão da organização funcional de áreas corticais. Em termos do que ocorre no cérebro, podemos falar em etapas de operações relacionadas ao processamento dos múltiplos sistemas de memória. Estas etapas são, respectivamente, codificação, consolidação e evocação e são bem conhecidas na literatura experimental com animais e com humanos (Zola-Morgan e Squire, 1990). A codificação refere-se às operaçōes de elaboração da informação para serem armazenadas. Geralmente envolve passagem por estruturas da região límbica do cérebro (McGaugh, 2000; Squire, Knowlton e Mussen, 1993; Markowitsch, 1995). A etapa de consolidação tem duração limitada e permite a retenção da informação para a elaboração e armazenamento. Envolve primordialmente vias da região temporal média, particularmente uma estrutura chamada hipocampo. Esta estrutura tem propriedade de processamento em conjunção com o córtex que permite a reverberação da informação ao ser armazenada. Esta ação de reverberação é essencial ao processo de consolidação. McGaugh (2000) faz uma revisão histórica dos achados sobre estas operaçôes de formação de memória à luz de dados das neurociências. Este artigo oferece evidências que refletem a fundamentação bem estabelecida no laboratório, na clínica e na teoria desta etapa na formação de memória.

A evocação é a etapa de acesso ao material armazenado. As vias cerebrais de evocação não são as mesmas onde estão armazenadas as informações (Markowitsch, 1995). Como descrevem Greenberg e Rubin (2003), apoiados por achados com neuroimagem funcional, a região primariamente responsável pela evocação é a região frontotemporal, diferente das regiōes do armazenamento, que ocorre em áreas corticais mais posteriores. Em relação à memória autobiográfica, a maioria dos autores concorda que as vias frontotemporais coordenam a ativação entre áreas extensas do córtex, produzindo um padrão de atividade neural semelhante àquele presente na ocasião da experiência original. Áreas extensas do neocórtex próximas aos córtices sensoriais provavelmente têm competência para armazenar informações, principalmente após muitas exposições aos dados, eventos, mas a extensão desta habilidade não é suficientemente conhecida (Kapur e Maclelland, 1992). Este padrão de ativação é bastante coerente com o conhecimento atual a respeito da organização cortical funcional. O lobo frontal e temporal tem função na associação complexa e na formação de novas memórias (Markowitsch, 1995), 
na consciência do eu (Knight e Grabowecky, 2000) e na auto-identidade (Keenan, Wheeler, Gallup Jr., e Pascual-Leone, 2000) e tem sido associado a distúrbios de personalidade (Raine, Lencz Bihrle, LaCasse e Colletti, 2000); portanto, nada mais justificado do que o recrutamento destas regiōes no processamento da evocação da história pessoal remota.

Outras propriedades neurocognitivas da região frontal também colaboram no sentido da individuação. $\mathrm{O}$ envolvimento da região frontal e suas conexões com o lobo temporal permitem a seletividade por inibição e ativação - um processamento crucial para o acesso aos padrôes de ativação cortical adequados à evocação de informações remotas do acervo pessoal. $\mathrm{O}$ gerenciamento do processamento pelas vias frontais coordena a experiência de evocação consciente de dados do passado autobiográfico como qualificado anteriormente, seletivamente acionando padrões relevantes e inibindo os irrelevantes.

Greenberg e Rubin (2003) acrescentam que a memória semântica é essencial para a evocação autobiográfica, graças à sua propriedade de integrar conhecimentos e formar laços com a simbologia particular. Apesar de as informações provavelmente estarem armazenadas em córtices sensoriais específicos, evidências com pacientes que apresentam demências semânticas sugerem que regiōes temporais laterais podem também armazenar informações semânticas.

Estudos com neuroimagem funcional mostraram um funcionamento assimétrico na ativação da região frontal nas operações de evocação. O lobo frontal esquerdo tem grande participação na organização da informação para a evocação tardia (Fletcher, Shallice, Frith, Frackowiak e Dolan, 1998), enquanto que a região homóloga contralateral parece ter relação com a forma pela qual uma determinada informação é evocada (Wheeler, Stuss e Tulving, 1997). De fato, Markowitsch (2003) documentou, em diversos estudos com neuroimagem, a importância da região frontotemporal direita na evocação de dados autobiográficos. Este autor afirma que a região lateral frontotemporal esquerda recebe informações semânticas, enquanto que a região contralateral é ativada durante a evocação de informações episódicas autobiográficas. Esta lateralização da evocação autobiográfica para o hemisfério direito provavelmente tem relação com a qualidade emocional da rememoração, visto a importância deste hemisfério em processos emocionais (Davidson e Irwin, 1999).

Vários autores têm investigado diferenças entre a evocação de informações negativas e positivas. Esta dimensão qualitativa acerca da valência emocional da informação evocada tem sido alvo de toda uma linha de pesquisa sobre neuropsicologia das emoções (Davidson e Irwin, 1999; Markowitsch, 2003; Frank e Tomaz, 2004). Sabe-se que a amígdala tem uma função peculiar na modulação 
emocional e no realce da informação para um armazenamento com as propriedades emocionais (Cahill e McGaugh, 1995; Adolphs, Cahill, Schul e Babinsky, 1997). Entretanto, ainda não está bem esclarecida a participação desta estrutura na evocação de informações autobiográficas. Alguns estudos indicam que a ativação da amígdala direita (Fink, Markowitsch, Reinkmeir, Bruckbauer, Kessler e Heiss, 1996) e esquerda (Lane, Reiman et al., 1997), entre outras estruturas, está envolvida na evocação de dados autobiográficos. A capacidade de modulação pode modificar a recuperação da memória autobiográfica, apesar de talvez não ter ação direta na operação de evocação. A amígdala tem conexões subcorticais com os córtices de associação incluindo região frontal, tendo, portanto, várias vias para exercer esta ação de realce da evocação autobiográfica. Como a evocação da informação envolve também uma reativação da rede neural formada na ocasião do processamento da informação, é possível que haja uma reativação da amígdala na etapa de rememoração.

A memória autobiográfica é rica em imagens, tendo, portanto, uma importante qualidade visual. Estudos com neuroimagem indicam que a evocação de uma história pessoal envolve a ativação da região occipital (Greenberg e Rubin, 2003), uma área especializada no processamento visual primário e secundário. A destruição do córtex occipital pode levar à amnésia retrograda. Portanto, esta região deve ser considerada quando buscamos definir as bases neurais da memória autobiográfica.

\section{NeUropsicologia dA AMNÉSIA RETRÓGRADA}

Técnicas modernas de neuroimagem vêem possibilitando o conhecimento das diferentes formas de amnésia e seus correlatos cerebrais. Estes avanços permitem esclarecer uma série de controvérsias entre amnésias orgânicas e amnésias psicogênicas, atualmente denominadas de amnésias dissociativas, ou seja, aquelas que ocorrem na ausência de uma lesão neural específica. Sabe-se hoje que estímulos estressantes presentes no meio ambiente podem prejudicar o funcionamento da memória graças a alteraçôes estruturais e químicas do cérebro e que a melhora da memória através da psicoterapia pode estar relacionada a ganhos no metabolismo cerebral. Por exemplo, Markowitsch (2003) descreveu o caso de um paciente que, após sofrer um trauma psíquico, apresentou amnésia retrograda e autobiográfica, manifestando também alterações no funcionamento padrão em determinadas regiốes neurais envolvidas na memória. Após tratamento psicológico bemsucedido da amnésia, o paciente voltou a apresentar os padrões esperados nestas regiōes neurais. 
Uma das explicações para os quadros de amnésia retrógrada, associados a um trauma e sem dano cerebral, seria a liberação alterada ou um desequilíbrio nos hormônios do eixo hipofisário-adrenal (glucocorticóides, mineralocorticóides) que atuariam bloqueando o processamento da memória autobiográfica (Markowitsch, 2003; McEwen, 2000). Nesse sentido, Anderson e colegas (2004) observaram que adultos, vítimas de abuso sexual na infância, manifestaram mudanças na morfologia cerebral assim como certos distúrbios de memória. Em referência ao estudo de Anderson, Markowitsch (2003) afirma que provavelmente alterações nos fatores de crescimento cerebral gerados pelo desequilíbrio destes neuro-hormônios causariam a mudança na morfologia cerebral. No mesmo artigo, Markowitsch, apoiado por pesquisas com neuroimagem de pacientes amnésicos, propóe que ambos os tipos de amnésia retrograda (orgânica ou dissociativa), envolvem processos dissociativos, gerando determinados bloqueios de memória. Este bloqueio ocorreria pela dessincronização do padrão de evocação. No caso da amnésia orgânica, seria devido a processos estruturais, enquanto que na amnésia dissociativa estariam envolvidas alteraçóes em processos neuroquímicos no nível da comunicação sináptica. Em ambos os casos é plenamente possível que este bloqueio surja da necessidade do eu de proteger-se de conteúdos ameaçadores. Esta concepção certamente tem relação com o conceito de mecanismos de defesa proposto por Freud. No entanto, outras noções advindas das teorias psicanalíticas ainda não encontraram respaldo na pesquisa da neuropsicologia da memória, como noções de simbologia pessoal relacionadas a conteúdo reprimido e o conceito de inconsciente como depositório desconhecido e potencialmente ameaçador. Cabe salientar que amnésia a é um fenômeno de esquecimento de informaçôes episódicas e da consciência. Envolve processos autonoéticos nos termos de Tulving (1987), ou seja, fenômenos em que existe uma consciência do engajamento do eu no processo de evocação.

\section{CONSIDERAÇÕES FINAIS}

A memória autobiográfica é uma forma complexa de memória com bases neurais próprias e distintas de outros tipos de memória. Esta qualidade única possivelmente decorre do caráter eminentemente auto-reflexivo da rememoração. Os estudos com neuroimagem têm identificado um padrão de ativação frontotemporal consistente na atividade de evocação do passado pessoal. Este padrão atribui à memória autobiográfica qualidades de processamento com configuração subjetiva dimensionada para aspectos emocionais específicos. O lobo frontal é uma estrutura importante nos processos relacionados a auto-identidade, consci- 
ência, e auto-regulação da emoção (Markowitsch, 2003; Fink et al., 1996; Keenan, Wheeler, Gallup e Pascual-Leone, 2000; Knight e Grabowecky, 2000). A região límbica contém vias altamente especializadas no processamento da emoção como na modulação emocional, no fortalecimento da memória e aprendizagem associativa com estímulos emocionais (Zola-Morgan e Squire, 1990; McGaugh, 2000; Cahill e McGaugh, 1995). A região orbitofrontal está relacionada com a motivação e principalmente com processos de auto-regulação avançados, sendo um local de convergência de informações extero e interoceptivas (Bechara, Tranel e Damásio, 2000). Estas propriedades funcionais permitem que a evocação autobiográfica seja seletiva com relação à expressão de uma história emocional, sujeita a efeitos de bloqueios gerados por mudanças neuro-hormonais e com variações de qualidade em função do estado motivacional do presente.

O direcionamento de processos de cima para baixo envolvidos na memória autobiográfica e a verticalidade da ativação das vias neurais na evocação deste sistema de memória ilustram outra dimensão significativa da relação entre conceito do eu e a neurocognição. Esta configuração do padrão da memória autobiográfica permite uma integração dinâmica entre o passado e o presente com as qualidades vivenciadas no passado, mas modificadas pela motivação no momento da evocação. A rede neural converge para uma região cerebral importante para a consciência do eu (região frontal), com o aporte de informações de vias de região temporal límbica e sublímbicas com conexôes somato-sensoriais e com áreas de processamento sensorial posteriores (lobo occipital no caso da memória autobiográfica). Isto oferece condições para a integração entre a rememoração do estado do corpo, a imagem visual e o conteúdo das lembranças com a auto-regulação proporcionada pelo estado emocional da pessoa no momento da evocação. Por exemplo, uma situação do passado vivida como uma ocasião festiva pode ser rememorada com pesar e com qualidades de culpa e dor psíquica num momento futuro, devido a um conflito interpessoal evoluído após o evento festivo - este pode ser o caso da recordação da cerimônia de casamento na fase da separação conjugal. Esta ação do presente sobre o passado pode ser bem mais sutil, como o esquecimento de partes dos fatos ou até mesmo confabulações que modificam os dados reais. Este padrão valida a importância da memória como um recurso importante na psicoterapia. Neste sentido, o processo de buscar re-vivenciar uma determinada experiência pessoal durante o atendimento psicanalítico configurase como uma verdadeira rememoração, incluindo aspectos sensoriais, viscerais, imagéticos e conceituais.

Entre as questôes para pesquisa na neuropsicologia, destacamos a investigação sobre a possibilidade de uma assimetria funcional em relação à evocação de 
fatos negativos (hemisfério direito) e positivos (hemisfério esquerdo). Outro aspecto em aberto na literatura é o envolvimento de região temporal mesial, particularmente o hipocampo, na etapa de evocação (Markowitsch, 1999, 2003). Dúvidas também estão presentes em termos do padrão de ativação na evocação de dados do passado remoto. Esta questão advém de problemas metodológicos e instrumentais. Isto porque as investigações através da imagem funcional ainda carecem da precisão temporal do registro necessária para realmente poder-se afirmar se as vias ativadas e as diferenças no metabolismo regional estão primariamente relacionadas ao processamento em questão ou se a ativação é um epifenômeno de uma rede cortical ainda desconhecida.

Finalmente, todos estes estudos indicam como a arquitetura cerebral foi sendo esculpida através de um processo de seleção natural, resultando em uma vida psíquica dinâmica, inter-relacionada com o meio interno e externo e com possibilidades de variações conjugadas. Aspectos neuroanatômicos e funcionais se entrecruzam para permitir esta riqueza da nossa atividade mental. Por exemplo, a disposição do sistema límbico em forma de anel formado por um conjunto de estruturas que evoluíram através da sofisticação progressiva de circuitos ao redor do tronco cerebral permite a interconexão mais econômica entre áreas corticais de associação. Possibilita também a comunicação entre áreas corticais especializadas com vias intermediárias de modulação sobre o aporte de informações subcorticais via hipotálamo e tálamo. Com a origem de estruturas corticais progredindo em direção às áreas neocorticais, surgiram também circuitos neurais capazes de comunicar-se entre si, o que possibilitou uma representação do meio externo e interno mais rica e precisa, assim como sua associação com aspectos emocionais. Mais ainda, este processo de evolução permitiu que mecanismos cognitivos pudessem alterar a forma pela qual estas informações previamente armazenadas podem ser evocadas. É nesta configuração que se insere a memória autobiográfica como um fenômeno de interação entre cognição e emoção relacionado à consciência do eu.

\section{REFERÊNCIAS BIBLIOGRÁFICAS}

Adolphs, R.; Cahill, L.; Schul, R.; Babinsky, R. (1997). Impaired declarative memory for emotional material following bilateral amygdala damage in humans. Learning and Memory, 4, 291-300. EUA.

Anderson, M. C.; Ochsner, K. N.; Kuhl, B.; Cooper, J.; Robertson, E.; Gabrieli, S. W.;

Glover, G. H.; Gabrieli, J. D. (2004). Neural systems underlying the suppression of 
unwanted memories. Science. Jan. 9, 2005, pp. 303 (5655), 232-5. EUA - Washington DC.

Baddely, A. (2000). Shorterm and working memory. Em Tulving, E. e Craik, F. M. (Orgs.). The Oxford Hanbook of Memory (pp. 77-92). Oxford University Press: Nova Iorque.

Bechara, A.; Tranel, D.; Damásio, H. (2000). Characterization of the decision-making deficit of patients with ventromedial prefrontal cortex lesions. Brain, 123, 2189-2202. Inglaterra, Oxford.

Cahill, L.; McGaugh, J. L. (1995). A novel demonstration of enhanced memory associated with emotional arousal. Conscious Cogn., 1995, Dec. 4 (4): 410-21.

Cowan, N. (1996). On short and long auditory stores. Psychological Bulletin, 16, 241-370.

Davidson, R. J. e Irwin, W. (1999). The functional neuroanatomy of emotion and affective style. Trends in Cognitive Science, 3, 11-21. Inglaterra, Londres.

De Renzi, E.; Lucchelli, F.; Muggia, S.; Spinnler, H. (1997). Is memory loss without anatomical damage tantamount to a psychogenic deficit? The case of pure retrograde amnesia. Neuropsychologia, 35, 781-794. EUA, Nova Iorque.

De Renzi, E. (2002). What does psychogen mean?. Cortex, 38, 678-681. Itália, Milão.

Dolan, R. J.; Lane, R.; Chua, P.; Fletcher, P. (2000). Dissociable temporal lobe activations during emotional episodic retrieval. NeuroImage, 11, 203-209. EUA, Nova Iorque.

Fink, G. R.; Markowitsch, H. J.; Reinkmeir, M.; Bruckbauer, T.; Kessler, J.; Heiss, W. D. (1996). Cerebral representation of one's own past: neural networks involved in autobiographical memory. Journal of Neuroscience, 16, 4275-4282. EUA, Washington DC.

Fletcher, P. C.; Shallice, T.; Frith, C. D.; Frackowiak, R. S.; Dolan, R. J. (1998). The functional roles of prefrontal cortex in episodic memory. II Retrieval. Brain, 121, 12491256. Inglaterra, Londres.

Frank, J. (2002). Tese de doutorado: Processamento emocional em pacientes submetidos a lobectomia temporal unilateral: contribuição ao estudo da memória emocional. Instituto de Biologia. Universidade de Brasília, DF, Brasília, Brasil.

Frank, J.; Tomaz, C. (2004). Lateralized impairment of the emotional enhancement of verbal memory in patients with amygdala-hippocampus lesion. Brain and Cognition, 52, 223-230. EUA, Nova Iorque.

Greenberg, D. L.; Rubin, D. C. (2003). The neuropsychology of autobiographical memory. Cortex, 2003, Sep-Dec, 39 (4-5), 687-728.

Halligan, P. W.; David, A. S. (2001). Cognitive neuropsychiatry towards a scientific psychopathology. National Review in Neuroscience, 2, 209-215. Inglaterra, Londres.

Kapur, N.; Ellison, D.; Smith, M. P.; Mclelland, D. L.; Burrows, E. H. (1992). Focal retrograde amnesia following bilateral temporal lobe pathology. A neuropsychological and magnetic resonance study. Brain, 115, 73-85. Inglaterra, Oxford. 
Keenan, J. P.; Wheeler, M.; Gallup Jr., G. G.; Pascual-Leone, A. (2000). Self-recognition and the right prefrontal cortex. Trends in Cognitive Sciences, 4, 338-344. EUA, Washington DC.

Knight, R. T.; Grabowecky, M. (2000). Prefrontal cortex, time and consciousness. Em Gazzaniga, M. S. (ed.) The New Cognitive Neurosciences (pp. 1319-1339), 2a ed. Cambridge, The MIT Press: EUA, Boston.

Lane, R. D.; Reiman, E. M.; Bradley, M. M.; Lang, P. G.; Ahern, G. L.; Davidson, R. J.; Schwartz, G. E. (1997). Neuroanatomic correlates of pleasant and unpleasant emotion. Neuropsychologia, 35, 1437-1444. EUA, Nova Iorque.

Markowitsch, H. J. (1995). Anatomical basis of memory disorders. Em Gazzaniga, M. S. (Org.). The Cognitive Neurosciences (pp. 765-779). Cambridge, The MIT Press: EUA, Boston.

. (1998/99). Differential contribution of the right and left amygdala to affective information processing. Behavioral Neurology, 11, 233-244. Holanda, Amsterdã.

-. (2003). Psychogenic amnesia. NeuroImage, 20, S132-S138.

McEwen, B. S. (2000). The neurobiology of stress from serendipity to clinical relevance. Brain Research, 886, 172-189. EUA, Nova Iorque.

McGaugh, J. (2000). Memory - a century of consolidation. Science, 287, 248-251. EUA, Washington DC.

Raine, A.; Lencz, T.; Birhle, S.; LaCasse, L.; Colletti, P. (2000). Reduced prefrontal gray matter volume and reduced autonomic activity in antisocial personality disorder. Archives of General Psychiatry, 57, 119-127. EUA, Nova Iorque.

Squire, L. R.; Knowlton, B.; Mussen, G. (1993). The structure and organization of memory. Annual Review of Psychology, 44, 453-495. EUA, Washington DC.

Tulving, E. (1987). Multiple memory systems and consciousness. Human neurobiology, 6, 67-70. Alemanha, Heidelberg.

Wheeler, M. A.; Stuss, D. T.; Tulving, E. (1997). Toward a theory of episodic memory: the frontal lobes and autonoetic consciousness. Psychological Bulletin, 121, 331-354. EUA, Washington DC.

Zola-Morgan, S.; Squire, L. R. (1990). The neuropsychology of memory. Parallel findings in humans and nonhumans primates. Annals of the Academy of Science of New York, 608, 434-456. EUA, Washington DC.

Recebido em 8 de fevereiro de 2006 Aceito para publicação em 16 de março de 2006 